\title{
Broadband corner cloak using a uniaxial transformation medium of stacked artificial dielectric sheets
}

\author{
Yuma Takano ${ }^{*}$ and Atsushi Sanada \\ Graduate School of Engineering Science, Osaka University, 1-3 Machikaneyama, Toyonaka, Osaka 560-8531, Japan
}

Received: 8 January 2020 / Accepted: 6 September 2020

\begin{abstract}
We demonstrate corner cloak operations mimicking a corner reflector and hiding objects in a truncated corner. The corner cloak is designed at $18.25 \mathrm{GHz}$ and implemented by nonresonant artificial dielectric sheets stacked onto the bottom hypotenuse. It is shown by the near-field measurements that the measured field distributions for the cloak agree well with those for the original area of the corner reflector as well as those for the numerical prediction. The bistatic radar cross-sections (BRCSs) for the cloak and the original area calculated from the measured field distributions coincide with each other and the cloak operation is quantitatively confirmed. The bandwidth evaluated by the specular scattering angles from the BRCSs shows broadband operation as wide as from 16 to $22 \mathrm{GHz}$.
\end{abstract}

Keywords: Transformation electromagnetics / invisibility cloaks / metamaterials

\section{Introduction}

The concept of transformation electromagnetics has been proposed, in which electromagnetic waves are arbitrarily controlled under coordinate transformations [1]. Based on the concept, transmission type cloaks have been introduced so that electromagnetic waves make a detour around spherical or cylindrical objects [2-4], followed by experimental demonstrations $[5,6]$. Reflection type cloaks, or socalled carpet cloaks, mimicking a flat floor have also been proposed to hide objects on a floor under a carpet [7-11], and experimentally demonstrated [12-25]. The spaces under consideration in these transmission and reflection type cloaks concepts are an infinite space and a semiinfinite space, respectively. As an extension, the concept of a corner cloak mimicking a corner reflector considering a quarter infinite space has been theoretically presented to hide objects in a truncated corner [26,27]. However, the concept has not been experimentally demonstrated.

An isovolumetric transformation (so-called area-preserving or unimodular transformation) over one of the walls [27-29] has been introduced in the corner cloak. Since the transformation yields a spatially constant material parameters with unity permeability in contrast to general coordinate transformations including [26] with spatially variant parameters and nonunity permeability, the corner cloak can be implemented by a homogeneous nonmagnetic

\footnotetext{
* e-mail: y-takano@ec.ee.es.osaka-u.ac.jp
}

material, which can lower the technological hurdle in implementations. In fact, the cloak is uniaxially anisotropic and thus can be easily implemented by rotating layered dielectric structures.

In this paper, the corner cloak hiding objects in a truncated corner is demonstrated. The corner cloak is designed based on an isovolumetric transformation and implemented using a uniaxial transformation medium of uniformly stacked nonresonant artificial dielectric sheets with intrinsically broadband characteristics. The uniaxial transformation medium is designed at $18.25 \mathrm{GHz}$ and implemented by artificial dielectric sheets stacked with the angle of the bottom hypotenuse. Near-field measurements are carried out to confirm cloak operations to hide a right triangle region. The bistatic radar cross-sections (BRCSs) are calculated from the measured field distributions to quantitatively verify the proposed concept. The bandwidth of the cloak is evaluated in terms of the specular scattering angles from the BRCSs.

\section{Corner cloak using uniaxial media}

Let us consider a 2D TM wave propagating in the first quadrant space shown in Figure 1a and apply an isovolumetric transformation [27] $\left(x^{\prime}, y^{\prime}\right) \rightarrow(x, y)$ with

$$
x=x^{\prime}, y=y^{\prime}-\frac{h}{a} x^{\prime}+h .
$$

This transformation creates a triangular space in the bottom left corner as shown in Figure $1 \mathrm{~b}$ and works as a 


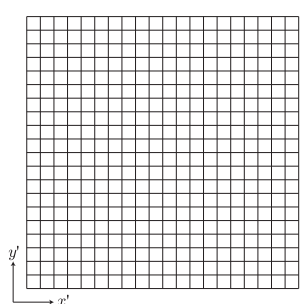

(a)

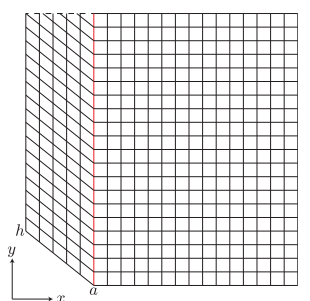

(b)
Fig. 1. Isovolumetric transformation. (a) Original coordinate system of the first quadrant space. (b) Transformed coordinate system creating the triangular space in the bottom left corner.

corner cloak hiding objects in the triangular space, leading to a corner reflector operation.

According to the transformation electromagnetics concept [1], the material parameters equivalent to the coordinate transformation are given as

$$
\overline{\bar{\varepsilon}}_{r}=\left(\begin{array}{cc}
1 & -p \\
-p & 1+p^{2}
\end{array}\right), \mu_{r}^{z}=1,
$$

where $p=h / a$. The above formulas indicate that the transformation medium can be realized by a nonmagnetic uniaxial medium having the principal permittivities of

$$
\varepsilon_{ \pm}=\frac{2+p^{2} \pm p \sqrt{p^{2}+4}}{2}
$$

with an appropriate rotation angle $\alpha$ around the $z$-axis:

$$
\alpha=\tan ^{-1}\left(-\frac{\sqrt{p^{2}+4}-p}{2}\right) .
$$

\section{Implementation}

The uniaxial medium is implemented by using artificial dielectric sheets each of which consists of a dielectric substrate with metal patches on both sides. We choose the inclination $p=1 / \sqrt{2}$ so that the rotation angle $\alpha$ coincides with the inclination angle $\theta=\tan ^{-1}(-p)$ of the bottom hypotenuse. In this case, the medium can be constructed with artificial dielectric sheets stacked onto the bottom hypotenuse, which leads to easy fabrication.

The artificial dielectric sheet is implemented by an array of metallic patches on a dielectric substrate. According to equation (3), the diagonalized permittivity tensor should be

$$
\operatorname{diag}\left(\varepsilon_{+}, \varepsilon_{-}\right)=\operatorname{diag}\left(2, \frac{1}{2}\right) .
$$

Instead of the above tensor with the smaller component less than one, we choose to realize a permittivity tensor of $\operatorname{diag}\left(\varepsilon_{+}^{\prime}, \varepsilon_{-}^{\prime}\right)=\varepsilon_{\mathrm{s}} \operatorname{diag}(2,1 / 2)$ with a scaling factor $\varepsilon_{\mathrm{s}}(\geq 2)$ to keep propagation directions at the cost of a little reflection. The artificial dielectric sheet is designed by using the finite element method based HFSS simulator (Ansys).

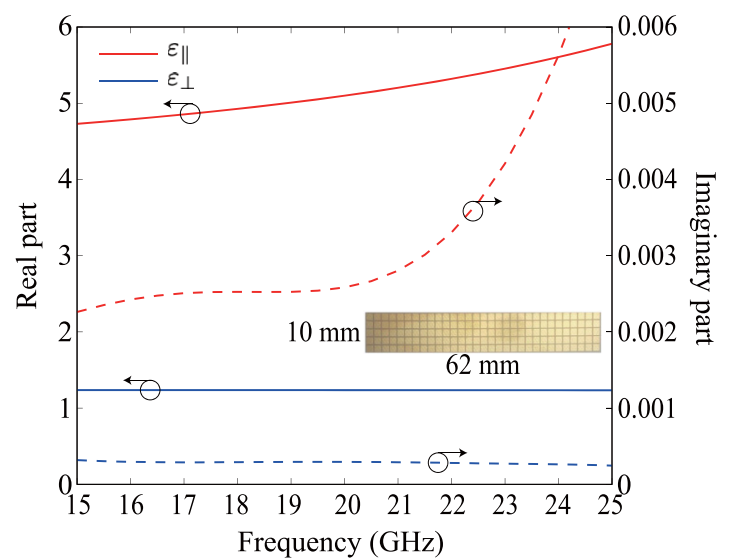

Fig. 2. Retrieved permittivity (simulation). The red solid and dashed lines represent the real and imaginary parts of $\varepsilon_{\|}$, respectively. The blue lines represent those for $\varepsilon_{\perp}$. The design frequency is $18.25 \mathrm{GHz}$. Inset: photograph of the designed artificial dielectric sheet.

The sheet consists of the dielectric substrate (relative permittivity 2.19, dielectric loss tangent 0.0006 , thickness $0.508 \mathrm{~mm}$ ) filled with square metal patches with the side $1.7 \mathrm{~mm}$ in every $2 \mathrm{~mm}$ on both sides (see the inset in Fig. 2).

In the simulations, the 2D Bloch-Floquet periodic boundary conditions are applied. The sheet exhibits a uniaxial anisotropy with the $c$-axis perpendicular to the sheet. Figure 2 shows the retrieved in-plane and perpendicular effective relative permittivities, $\varepsilon_{\|}$and $\varepsilon_{\perp}$, for the case where the sheets are stacked with the period $1.6 \mathrm{~mm}$. It is seen from Figure 2 that the permittivity tensor is realized at $18.25 \mathrm{GHz}$ with the scaling factor $\varepsilon_{\mathrm{s}}$ of $1.58^{2}$ having a moderate frequency dependence. The imaginary parts for $\varepsilon_{\|}$and $\varepsilon_{\perp}$ are $2.5 \times 10^{-3}$ and $3.0 \times 10^{-4}$, which correspond to the quality factors of 1980 and 4130 , respectively.

One hundred and twenty designed sheets of $62 \mathrm{~mm}$ $\times 10 \mathrm{~mm}(31 \times 5$ cells $)$ are fabricated using the doublesided lithography technique for printed circuit boards to hide a right triangle region with $a=50 \mathrm{~mm}$ and $h=a / \sqrt{2}(\approx 35.56 \mathrm{~mm})$. The sheets are supported with the designed inclination of $p=1 / \sqrt{2}$ and the designed period of $1.6 \mathrm{~mm}$ by a $3 \mathrm{D}$ printed jig.

\section{Experiments}

In order to confirm the operation of the corner cloak, $2 \mathrm{D}$ near-field measurements are carried out. Figure 3 shows the measurement system. The cloak is placed on a foam support with a height of $280 \mathrm{~mm}$ on a metallic platen. Two metallic plates are placed on the bottom hypotenuse and the left side of the cloak in Figure 3. The plates' sizes are limited to be 62 and $50 \mathrm{~mm}$, respectively, to suppress higher order scatterings and emphasize the specular (zeroth order) scattering. The cloak is illuminated by a K-band standard horn antenna with the TM incidence. The complex electric near-field distributions on the plane $0.1 \mathrm{~mm}$ above the cloak are measured with a vector network analyzer. A cylindrical waveguide probe controlled by an 
automated $x y z$-stage is used to pick up in-plane electric fields. The total measurement area is $150 \mathrm{~mm} \times 150 \mathrm{~mm}$ with the sampling period of $2 \mathrm{~mm} \times 2 \mathrm{~mm}$. Oblique incident waves with the angle $\theta_{\text {inc }}$ of $0,30^{\circ}, 45^{\circ}$, and $60^{\circ}$ are applied by changing the position of the horn antenna.

Figure $4 \mathrm{a}$ shows the measured field distributions at the target frequency of $18.3 \mathrm{GHz}$ in the case of $\theta_{\text {inc }}=0^{\circ}$ with those for the original area and for an area with a slope

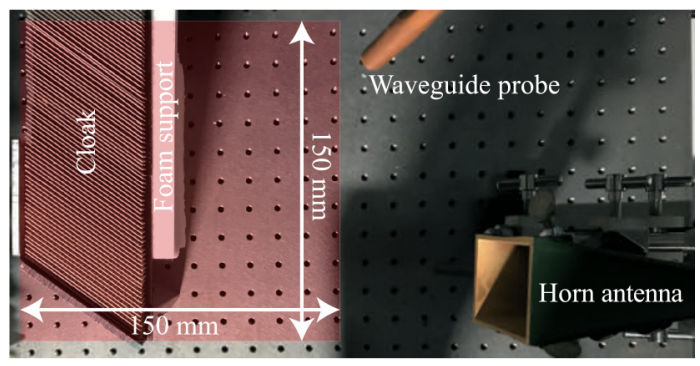

Fig. 3. Top view of the measurement system. The measured area is colored in red.
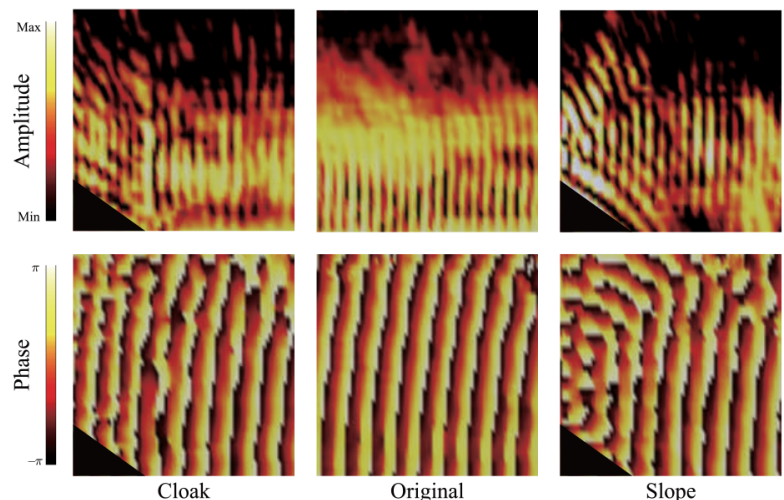

(a) without the cloak as comparisons. It is seen from the figure that the incident wave propagates according to the coordinate system in the cloak and is reflected back to the direction of the incidence leading to a standing wave in the $x$-direction, which agrees well with the measured field distribution for the original area. In addition, by comparing the measured field distribution for the cloak with that for a slope, it is seen that the specularly reflected wave by the slope (in the theoretical direction of $70.5^{\circ}$ ) is significantly suppressed by the cloak. Figure $4 \mathrm{~b}$ shows the corresponding field distributions simulated based on the equivalent circuit model of full-tensor 2D anisotropic metamaterials [30]. It is clearly seen from the figure that the simulated results well indicate the measured phenomena; the wave propagates according to the coordinate system with little specular reflection by the slope, leading to the validity of the measured results. As another example, Figure 5 a shows the measured field distributions in the case of $\theta_{\text {inc }}=60^{\circ}$. It is seen from the figure that the system operates as a corner reflector thanks to the corner cloak and the incident wave is reflected back toward the direction of arrival of $60^{\circ}$, which

Fig. 4. Measured and simulated field distributions for the incident angle of $\theta_{\text {inc }}=0^{\circ}$ (@18.3 GHz). (a) Measured results. (b) Simulated results by the circuit simulations. The upper and lower rows represent complex amplitude and phase distributions, respectively. The left, center, and right columns represent field distributions for the cloak, the original area, and the slope, respectively.
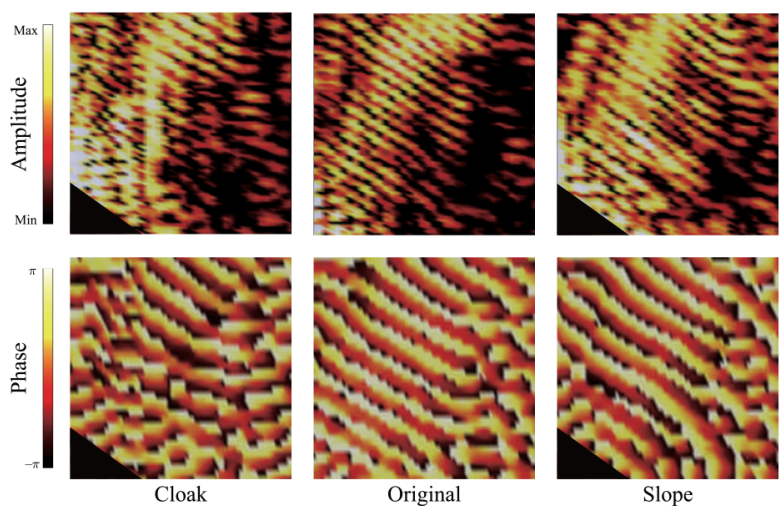

(a)

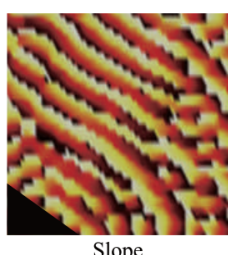

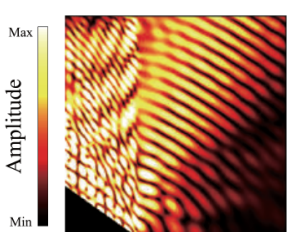
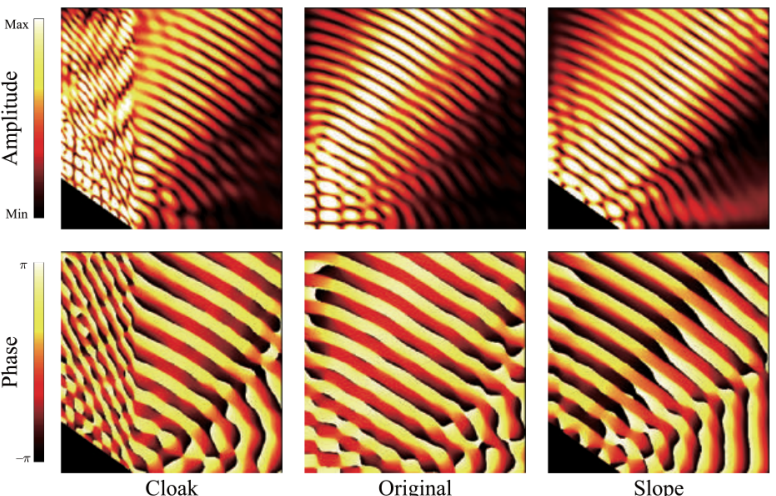

(b)

Fig. 5. Measured and simulated field distributions for the incident angle of $\theta_{\text {inc }}=60^{\circ}$ (@18.3 GHz). (a) Measured results. (b) Simulated results by the circuit simulations. The upper and lower rows represent complex amplitude and phase distributions, respectively. The left, center, and right columns represent field distributions for the cloak, the original area, and the slope, respectively. 


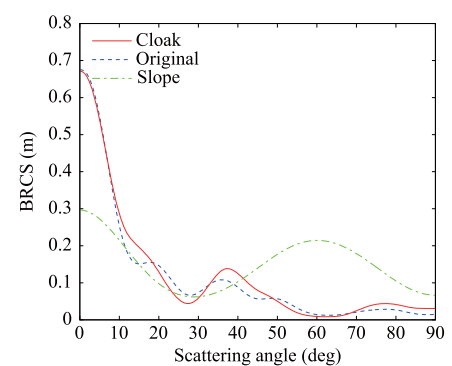

(a)

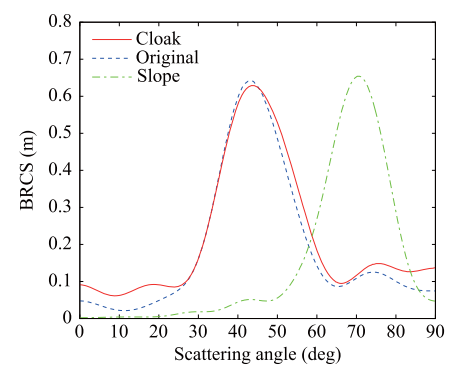

(c)

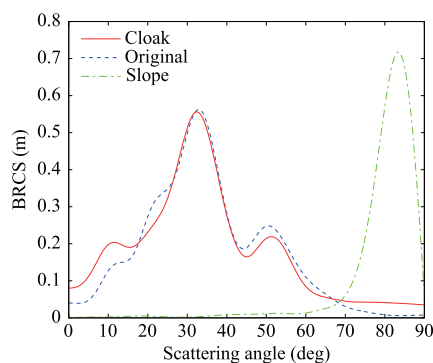

(b)

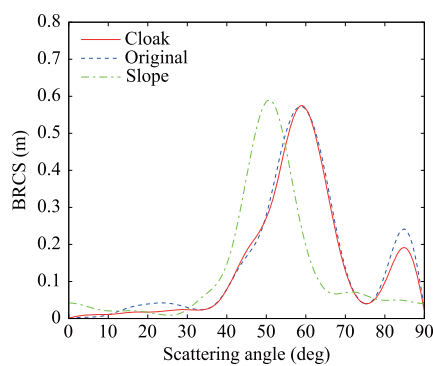

(d)
Fig. 6. BRCSs calculated from the measured field distributions for the incident angles of (a) $\theta_{\text {inc }}=0^{\circ}$, (b) $\theta_{\text {inc }}=30^{\circ}$, (c) $\theta_{\text {inc }}=45^{\circ}$, and (d) $\theta_{\text {inc }}=60^{\circ}$. In each figure, the red solid line is for the cloak, the blue dashed line is for the original area and the green dotdashed line is for the slope.

agrees well with the measured field distribution for the original area. On the other hand, in the case with a slope without the cloak, most of the incident wave is specularly reflected by the slope in the direction of slightly smaller angle than $60^{\circ}$ (theoretically $49.5^{\circ}$ ) resulting in the broaden standing wave distribution. The corresponding simulated results shown in Figure 5b clearly illustrates the phenomena and support the measured results as well. Based on the foregoing discussion, the corner cloak operation is qualitatively confirmed. Incidentally, the electromagnetic field in the transformed region can include scattered waves from the top boundary of the cloak due to the finite implementation, however this scattering would affect less on the cloak operation as the top boundary becomes sufficiently far from the beam.

In order to quantitatively confirm the cloak operation, BRCSs at the target frequency of $18.3 \mathrm{GHz}$ are calculated from the measured field distributions. The calculated BRCSs are shown in Figure 6 for the incident angles of (a) $\theta_{\text {inc }}=0^{\circ}$, (b) $\theta_{\text {inc }}=30^{\circ}$, (c) $\theta_{\text {inc }}=45^{\circ}$, and (d) $\theta_{\text {inc }}=60^{\circ}$. In the figures, the red solid lines are for the cloak, the blue dashed lines are for the original area and the green dotdashed lines are for the slope. According to Figure 6a, the BRCS for the cloak agrees well with that for the original area and has the peak at $0^{\circ}$, which suggests that most of the incident power is reflected back toward the source direction. The difference between these two BRCSs is considered to be mainly due to the scaling factor $\varepsilon_{\mathrm{s}}=1.58^{2}$ introduced in the cloak design. On the other hand, the BRCS for the slope has smaller peaks at $0^{\circ}$ and $61^{\circ}$ which would result from scatterings by the two metallic plates. According to similar results in Figures $6 \mathrm{~b}-6 \mathrm{~d}$, the BRCSs for the cloak and the original area agree well with each

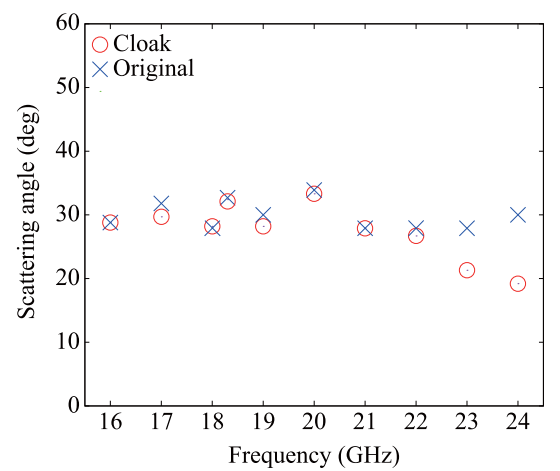

Fig. 7. Frequency dependence of the specular scattering angles obtained from the BRCSs for $\theta_{\text {inc }}=30^{\circ}$. The red circles represent the scattering angles for the cloak and the blue cross marks represent those for the original area.

other with the differences between the specular scattering angles less than $0.5^{\circ}$. Incidentally, the angle differences between the specular scattering angles $\left(32.4^{\circ}, 43.8^{\circ}\right.$, and $\left.59.1^{\circ}\right)$ and the target incident angles $\left(30^{\circ}, 45^{\circ}\right.$, and $\left.60^{\circ}\right)$ are considered to be due to the radiation characteristics of the horn antenna. In addition, it is seen from the figures that the specular scatterings for the slope in $83.4^{\circ}, 69.3^{\circ}$, and $50.7^{\circ}$ are significantly suppressed by the cloak. Based on these results, the angle independent cloak operation is quantitatively confirmed.

We also investigate the frequency dependence of the specular scattering angles. Figure 7 shows the measured specular scattering angles at 16-24 GHz band in the case of $\theta_{\text {inc }}=30^{\circ}$. It is seen from Figure 7 that the differences between the scattering angles for the cloak and the original area are less than $0.5^{\circ}$ below $23 \mathrm{GHz}$. This reflects the dispersion characteristics of the medium in Figure 2; i.e., $\varepsilon_{\|}$ significantly increases as the frequency becomes high and closer to the resonant frequency, whereas $\varepsilon_{\|}$approaches to a constant value as the frequency decreases. Consequently, the broadband operation of the proposed cloak is experimentally shown. Incidentally, the fluctuation of the specular scattering angles with the frequency would result from the radiation characteristics of the incident horn antenna.

\section{Conclusions}

In conclusion, the corner cloak operation hiding objects in the triangular region in the corner has been demonstrated. The corner cloak has been designed and implemented using a uniaxial transformation medium of uniformly stacked nonresonant artificial dielectric sheets with intrinsically broadband characteristics. The design formulas for the principle permittivities and the rotation angle have been shown. The uniaxial transformation medium has been designed at $18.25 \mathrm{GHz}$ and implemented by 120 artificial dielectric sheets of $62 \mathrm{~mm} \times 10 \mathrm{~mm}$ stacked with the angle of the bottom hypotenuse. Cloak operations to hide a right triangle region with the base of $50 \mathrm{~mm}$ and the height of $35.36 \mathrm{~mm}$ have been demonstrated with incident waves having several incident angles. The measured field 
distributions agree well with the predicted results by simulations. The BRCSs calculated from the measured field distributions for the cloak and the original area agree well with each other with the differences between the specular scattering angles less than $0.5^{\circ}$ and the validity of the proposed concept has been experimentally shown. The bandwidth evaluated by the specular scattering angles from the BRCSs shows broadband operation at least from 16 to $22 \mathrm{GHz}$.

This work was partially supported by JSPS KAKENHI Grant Number 19J20104.

\section{References}

1. J.B. Pendry, D. Schurig, D.R. Smith, Science 312, 1780 (2006)

2. S.A. Cummer, B.I. Popa, D. Schurig, D.R. Smith, J. Pendry, Phys. Rev. E 74, 036621 (2006)

3. W. Cai, U.K. Chettiar, A.V. Kildishev, V.M. Shalaev, Nat. Photon. 1, 224 (2007)

4. W. Cai, U.K. Chettiar, A.V. Kildishev, V.M. Shalaev, G.W. Milton, Appl. Phys. Lett. 91, 111105 (2007)

5. D. Schurig, J.J. Mock, B.J. Justice, S.A. Cummer, J.B. Pendry, A.F. Starr, D.R. Smith, Science 314, 977 (2006)

6. B. Kanté, D. Germain, A. de Lustrac, Phys. Rev. B 80, 201104 (2009)

7. J. Li, J.B. Pendry, Phys. Rev. Lett. 101, 203901 (2008)

8. Z. Chang, X. Zhou, J. Hu, G. Hu, Opt. Express 18, 6089 (2010)

9. N.I. Landy, N. Kundtz, D.R. Smith, Phys. Rev. Lett. 105, $193902(2010)$

10. M.A.F.C. Junqueira, L.H. Gabrielli, D.H. Spadoti, J. Opt. Soc. Am. B 32, 2488 (2015)

11. D.G. Silva, P.A. Teixeira, L.H. Gabrielli, M.A.F.C. Junqueira, D.H. Spadoti, Opt. Express 25, 23517 (2017)
12. L.H. Gabrielli, J. Cardenas, C.B. Poitras, M. Lipson, Nat. Photon. 3, 461 (2009)

13. J. Valentine, J. Li, T. Zentgraf, G. Bartal, X. Zhang, Nat. Mater. 8, 568 (2009)

14. R. Liu, C. Ji, J.J. Mock, J.Y. Chin, T.J. Cui, D.R. Smith, Science 323, 366 (2009)

15. J.H. Lee, J. Blair, V.A. Tamma, Q. Wu, S.J. Rhee, C.J. Summers, W. Park, Opt. Express 17, 12922 (2009)

16. H.F. Ma, W.X. Jiang, X.M. Yang, X.Y. Zhou, T.J. Cui, Opt. Express 17, 19947 (2009)

17. T. Ergin, N. Stenger, P. Brenner, J.B. Pendry, M. Wegener, Science 328, 337 (2010).

18. H.F. Ma, T.J. Cui, Nat. Commun. 1, 21 (2010)

19. J. Zhang, L. Liu, Y. Luo, S. Zhang, N.A. Mortensen, Opt. Express 19, 8625 (2011)

20. M. Gharghi, C. Gladden, T. Zentgraf, Y. Liu, X. Yin, J. Valentine, X. Zhang, Nano Lett. 11, 2825 (2011)

21. X. Chen, Y. Luo, J. Zhang, K. Jiang, J.B. Pendry, S. Zhang, Nat. Commun. 2, 176 (2011)

22. B. Zhang, Y. Luo, X. Liu, G. Barbastathis, Phys. Rev. Lett. 106, 033901 (2011)

23. J. Fischer, T. Ergin, M. Wegener, Opt. Lett. 36, 2059 (2011)

24. D. Shin, Y. Urzhumov, Y. Jung, G. Kang, S. Baek, M. Choi, H. Park, K. Kim, D.R. Smith, Nat. Commun. 3, 1213 (2012)

25. M.H. Fakheri, A. Abdolali, S. Hashemi, B. Noorbakhsh, Microwave Opt. Technol. Lett. 59, 1284 (2017)

26. W. Zhu, I. Shadrivov, D. Powell, Y. Kivshar, Opt. Express 19, $20827(2011)$

27. Y. Takano, A. Sanada, in Proceedings of2018 48th European Microwave Conference, 2018, pp. 679-682

28. S. Xu, H. Xu, H. Gao, Y. Jiang, F. Yu, J.D. Joannopoulos, M. Soljačić, H. Chen, H. Sun, B. Zhang, Proc. Natl. Acad. Sci. U.S.A. 112, 7635 (2015)

29. B. Orazbayev, M. Beruete, A. Martnez, C. Garca-Meca, Phys. Rev. A 94, 063850 (2016)

30. T. Nagayama, A. Sanada, IEEE Trans. Microwave Theory Tech. 63, 3851 (2015)

Cite this article as: Yuma Takano, Atsushi Sanada, Broadband corner cloak using a uniaxial transformation medium of stacked artificial dielectric sheets, EPJ Appl. Metamat. 7, 4 (2020) 